\title{
The measurement problem in Quantum Mechanics: Convivial Solipsism
}

\author{
Hervé Zwirn ${ }^{1}$
}

\begin{abstract}
The problem of measurement is often considered an inconsistency inside the quantum formalism. Many attempts to solve (or to dissolve) it have been made since the inception of quantum mechanics. The form of these attempts depends on the philosophical position that their authors endorse. I will review some of them and analyze their relevance. In this paper, I defend a new position, the "Convivial Solipsism", according to which the outcome that is observed is relative to the observer, different but in close parallel to the Everett's interpretation and sharing also some similarities with Rovelli's relational interpretation and Quantum Bayesianism. I also show how "Convivial Solipsism" can help getting a new standpoint about the EPR paradox providing a way out of the seemingly unavoidable non-locality of quantum mechanics.
\end{abstract}

Keywords Measurement problem, consciousness, decoherence, realism, entanglement, non-locality

\section{Introduction}

La mécanique quantique est l'une des théories les plus précises dont nous disposions actuellement en physique. Elle permet de comprendre un très grands nombre de phénomènes qui vont de la structure de l'atome à la couleur des corps, en passant par la supraconductivité. Elle fonctionne remarquablement bien et n'a jamais été mise en défaut depuis quasiment un siècle qu'elle a été inventée. Cependant, si on cherche à analyser les fondements mathématiques sur lesquels elle repose, on se heurte à des difficultés d'interprétations qui opposent les physiciens depuis sa naissance même. Le problème ne réside pas dans l'utilisation de son formalisme pour calculer le comportement d'un système ou pour prédire le résultat d'une expérience. Dans ce domaine, tous les physiciens s'accordent sur une manière commune de procéder et qui fonctionne très bien. La question est plutôt de savoir ce que représentent réellement les entités mathématiques qui constituent son formalisme. Par exemple, les outils qu'on appelle fonction d'onde ou vecteur d'état représentent-ils vraiment l'état d'un système réel ou ne sont-ils que des intermédiaires de calculs qui permettent de prédire le résultat numérique de telle ou telle expérience ? Autre possibilité : représentent-ils en fait uniquement la connaissance qu'un physicien a du système et non pas le système en lui-même ? Lorsqu'un physicien constate le résultat d'une mesure, ce résultat matérialise-t-il un véritable changement dans l'état du système ou n'est-il que la mise à jour de la connaissance du physicien à propos de cet état ? En fait, dès qu'on s'interroge plus précisément sur ce type de questions, on ouvre la porte à une série de problèmes qui soulèvent des difficultés actuellement non résolues de manière consensuelle. En particulier, un point important est de décider si la mécanique quantique décrit la réalité telle qu'elle est vraiment ou si, malgré son succès empirique, elle n'est qu'une suite de recettes efficaces pour prédire des résultats expérimentaux. La question du statut de ce qu'on appelle une observation est centrale dans l'étude des fondements de la mécanique quantique. Contrairement à ce qui se passe en physique classique ou le concept d'observation ne pose aucun problème, la mécanique quantique interdit de considérer qu'une observation n'est rien d'autre que le constat passif par un observateur d'une situation préexistante. Il semble que ce soit le fait même de mesurer les propriétés d'un système qui soit à l'origine de la valeur que prennent ces propriétés. Cela est bien entendu extrêmement contre-intuitif et demande à être expliqué plus précisément. Or, la difficulté principale est l'impossibilité dans laquelle se trouve la mécanique quantique de donner à l’intérieur même de son formalisme une définition précise et objective de ce qu'est une mesure. C’est

\footnotetext{
${ }^{1}$ UFR de Physique (LIED, Université Paris 7), CMLA (ENS Cachan, France) \& IHPST (CNRS, France), herve.zwirn@gmail.com
} 
ce qu'on appelle le "problème de la mesure » et c'est à ce sujet que nous allons consacrer ce texte. Comme nous le verrons, résoudre le problème de la mesure va nous amener à analyser des suggestions très variées qui ont été faites au cours de l'histoire et à proposer une solution qui remet profondément en cause la notion de la réalité que nous pouvons avoir selon le sens commun.

\section{Le problème de la mesure}

Le problème de la mesure vient du fait que le formalisme quantique contient deux postulats différents pour calculer l'évolution d’un système. Le premier est l'équation de Schrödinger :

$$
i \hbar \frac{d|\Psi\rangle}{d t}=H|\Psi\rangle
$$

qui est supposée être utilisée pour décrire l'évolution d'un système isolé quand aucune mesure n'est faite sur lui. Le second est le postulat de réduction du paquet d'onde qui traite de la mesure d'une certaine observable $\mathrm{P}$ sur un système qui est initialement dans un état de superposition des états propres de $\mathrm{P}$, $|\Psi\rangle=\sum c_{i}\left|\varphi_{i}\right\rangle$. Dans ce cas, si le résultat de la mesure est $\lambda_{\mathrm{k}}$, une des valeurs propres de P, l'état $|\Psi\rangle$ est projeté sur le vecteur propre $\left|\varphi_{k}\right\rangle$ lié à cette valeur propre, $\sum c_{i}\left|\varphi_{i}\right\rangle \rightarrow\left|\varphi_{k}\right\rangle$ ou sur le sous-espace engendré par les vecteurs propres liés à $\lambda_{\mathrm{k}}$, si $\lambda_{\mathrm{k}}$ est dégénérée.

L'application de ces deux principes ne donne pas les mêmes résultats. Si on suit la célèbre présentation de von Neumann [1], une mesure est une interaction entre un système $S$ dans un état $\left|\Psi_{S}\right\rangle=$ $\sum c_{i}\left|\varphi_{i}\right\rangle$ et un appareil $\mathrm{A}$ dans un état initial $\left|A_{0}\right\rangle$ à travers un hamiltonien d'interaction $\mathrm{H}_{\text {int }}$ agissant durant une coure durée. Avant qu'ils n’interagissent, l'état du système total constitué à la fois du système et de l'appareil est :

$$
\left|\Psi_{S A}\right\rangle=\left|\Psi_{S}\right\rangle \otimes\left|A_{0}\right\rangle=\sum c_{i}\left|\varphi_{i}\right\rangle \otimes\left|A_{0}\right\rangle
$$

Après l'interaction entre le système et l'appareil l'équation de Schrödinger qui décrit un processus linéaire et unitaire donne :

$$
\left|\Psi_{S A}\right\rangle=\sum c_{i}\left|\varphi_{i}\right\rangle \otimes\left|A_{0}\right\rangle \rightarrow \sum c_{i}\left|\varphi_{i}\right\rangle \otimes\left|A_{i}\right\rangle
$$

où $\left|A_{i}\right\rangle$ est l'état de l'appareil correspondant au résultat $\lambda_{\mathrm{i}}$. Maintenant si la valeur $\lambda_{\mathrm{k}}$ est trouvée (supposons qu'elle n'est pas dégénérée), le postulat de réduction donne :

$$
\left|\Psi_{S}\right\rangle=\sum c_{i}\left|\varphi_{i}\right\rangle \rightarrow\left|\varphi_{k}\right\rangle \text { et }\left|A_{0}\right\rangle \rightarrow\left|A_{k}\right\rangle
$$

L’équation (3) montre qu'après l'interaction, le system et l'appareil sont dans un état intriqué. En particulier, cela peut être interprété comme si l'appareil était dans une superposition d'états liés aux différents résultats possibles de la mesure ${ }^{2}$. Bien sûr, aucune superposition macroscopique de ce genre n’a jamais été observée. . Si on ajoute un autre système (comme un chat ou un homme) au système initial et à l'appareil, il s'intrique également avec les deux premiers. C'est le principe du fameux chat de Schrödinger [2] et de l'ami de Wigner [3].

Il serait possible de penser que la mécanique quantique ne s'applique qu'aux ensembles de systèmes et une interprétation facile serait alors qu'un état superposé représente un ensemble statistique d'états différents. Mais il est facile de montrer qu'une telle interprétation ne marche pas. Donc si nous considérons que la mécanique quantique est complète (c’est-à-dire, ne doit pas être complétée par des

\footnotetext{
${ }^{2}$ En fait, l'appareil étant dans un état intriqué avec le système ne possède pas d'état à proprement parler puisqu'à travers l'intrication seul le système composé $\mathrm{S}+\mathrm{A}$ a un état. Dire que l'appareil est dans une superposition d'état $\left|A_{i}\right\rangle$ est donc en toute rigueur faux. Le formalisme correct pour traiter ce type de situation est celui des matrices densité.
} 
variables cachées), une mesure ne peut pas être considérée comme un processus qui ne fait que révéler une valeur pré-existante.

Ceci montre que le problème de l'incompatibilité entre les deux principes d'évolution ne peut pas être résolu facilement. L'équation de Schrödinger décrit un processus unitaire tandis que le postulat de réduction ne l'est pas. Cela signifie qu'il n'y a a priori aucune possibilité d'obtenir une réduction par l'équation de Schrödinger. Ceci ne serait pas un problème s’il était possible de définir rigoureusement ce qu'est une mesure. Dans ce cas, le postulat de réduction s'appliquerait lors d'une mesure et on utiliserait l'équation de Schrödinger en l'absence de mesure.

Est-il possible de définir clairement ce qu'est une mesure ? Ce que beaucoup de physiciens cherchent est une définition objective, au sens où elle ne fait pas mention d'un observateur humain. Largement acceptée par beaucoup de physiciens qui imaginent qu'elle résout les problèmes, l'interprétation de Copenhague, avancée principalement par Bohr, dit qu'une mesure est simplement une interaction entre un système et un appareil de mesure macroscopique. Cette définition est-elle objective ? Nous allons voir qu'en dépit des apparences ce n’est pas le cas.

Nous passerons rapidement en revue plusieurs des principales solutions qui ont été avancées et tenterons de montrer en quoi elles sont insatisfaisantes. Nous exposerons alors le Solipsisme Convivial qui résout le problème de la mesure de manière satisfaisante même s'il conduit à une transformation assez radicale du concept de réalité auquel on est habitué.

\section{De nombreuses interprétations}

Face à ce problème qui ressemble à une véritable contradiction, les physiciens ont proposé de nombreuses solutions qui dépendent pour une grande part de leurs penchants philosophiques. Une description sommaire des différentes familles de position est la suivante :

- Les positions instrumentalistes considèrent que la mécanique quantique n'est qu'un outil permettant de prédire le résultat des mesures effectuées par les physiciens. Il ne faut donc pas la voir comme une description objective de la réalité. La position de Bohr et l'interprétation de Copenhague, défendue également par Heisenberg, sont instrumentalistes. Dans la même famille, les interprétations pragmatistes essaient de coller à la pratique des physiciens et acceptent comme entités de base des caractéristiques que d'autres interprétations essaient d'expliquer (comme par exemple l'unicité du résultat de mesure) [4-6]. Certaines positions pragmatistes sont cependant partiellement réalistes [7].

- Les positions réalistes considèrent qu'il y a une réalité extérieure, indépendante de tout observateur, qu'elle ressemble à peu près à ce que nous en percevons et que le rôle de la physique est d'en donner une description la plus fidèle possible. C'était la position spontanée de la majorité des physiciens au XIXième siècle et au début du XXième siècle et c'est dans ce cadre que la formulation initiale de la mécanique quantique a été faite. C'était la position d'Einstein, Schrödinger, Bell, de Broglie et Bohm.

- Les positions idéalistes donnent la primauté à l'esprit et considèrent que tout ce que nous percevons n'est rien d'autre qu'une création de notre esprit. Il existe de nombreux types d'idéalisme qui dépendent du degré auquel le concept de réalité est accepté. La forme la plus extrême est le solipsisme qui nie l'existence d'autre chose que l'esprit d'une seule personne (celle qui s'exprime). Comme nous le verrons, le Solipsisme Convivial n'est en fait pas un véritable solipsisme en ce qu'il accepte non seulement l'existence d'autres esprits mais également celle d'une réalité extérieure. Un idéalisme prudent peut se borner à affirmer que la seule réalité à laquelle nous avons accès est celle de nos perceptions et rester agnostique sur l'existence d'une réalité indépendante. L'idéalisme transcendantal de Kant ne nie pas l'existence d'une réalité (la chose en soi, les noumènes) mais prétend qu'il existe un fossé cognitif infranchissable entre les phénomènes que nous percevons et les noumènes qui ne sont pas connaissables. 
Il n’est évidemment pas question ici de passer en revue la totalité des interprétations qui ont été proposées pour résoudre le problème de la mesure. Même pour les plus récentes, à comparer avec le Solipsisme Convivial, je me contenterai de donner quelques indications sur les raisons pour lesquelles je ne les trouve pas satisfaisantes.

Je passerai rapidement sur les interprétations qui modifient le formalisme quantique et je me concentrerai sur celles qui se placent à l’intérieur du formalisme standard.

\subsection{Les interprétations qui modifient le formalisme quantique}

\subsubsection{Les theories à variables cachées}

Pendant de nombreuses années, une preuve donnée par von Neumann en 1932 a fait croire qu’il était impossible d'ajouter des variables cachées à la mécanique quantique. Mais Bell [8] réussit à construire une théorie de ce type pour un spin $1 / 2$ et l'exemple d'aujourd'hui est la théorie de Bohm [9] qui reproduit toutes les prédictions de la mécanique quantique en introduisant des variables cachées qui sont les positions des particules. Dans cette théorie, qui ajoute une seconde équation à l'équation de Schrödinger, le problème de la mesure n'existe pas car toute particule occupe à tout moment une position bien définie dans l'espace.

\subsubsection{Modification de l'équation de Schrödinger}

Comme nous l'avons vu, l'équation de Schrödinger décrivant une évolution déterministe, linéaire et unitaire ne peut rendre compte de la réduction probabiliste du paquet d'ondes. Il est donc naturel de tenter de la modifier pour lui ajouter un terme qui expliquerait cette réduction lorsqu'une mesure est faite mais qui préserverait les prédictions courantes en dehors de toute mesure. La plus connue des théories de ce type est celle de Ghirardi, Rimini and Weber (théorie GRW) [10] qui ajoute un terme non linéaire stochastique à l'équation de Schrödinger. La fonction d'onde est alors soumise à des localisations spontanées aléatoires qui, lorsque les paramètres sont correctement ajustés, permettent aux systèmes macroscopiques d'être bien localisés sans pour autant modifier les prédictions quantiques des micro-systèmes. Le problème de la mesure est donc là aussi résolu. Il existe des différences de prédictions avec la mécanique quantique mais elles ne sont pas testables avec les moyens actuels [11].

\subsubsection{Ces modifications sont-elles acceptables?}

Il n’est pas dans l'objet de cet article de détailler les différents problèmes posés par ces théories qui proposent un nouveau formalisme. Nous nous contenterons ici de dire qu'il semble qu'il soit difficile d'en construire une extension relativiste bien que des progrès aient été faits dans ce domaine ${ }^{3}$. Mais par ailleurs, ces théories ne sont pas exemptes de difficultés. La théorie GRW explique la réduction du paquet d'ondes par un mécanisme qui semble vraiment ad hoc. La théorie de Bohm était initialement destinée à restaurer une représentation intuitive des particules et de leur trajectoire, ce que la mécanique quantique interdit. Mais en fait, l'image de la réalité donnée par cette théorie est très loin de son but initial et le réalisme de la théorie de Bohm ne ressemble que très peu à celui de la mécanique classique. La question de décider si l'une de ces théories doit être acceptée est pour le moment ouverte mais aucune d'entre elles n'a réussi à obtenir un consensus en sa faveur ${ }^{4}$. Cela justifie l'effort de tenter de résoudre le problème de la mesure en conservant le cadre quantique standard.

\footnotetext{
${ }^{3}$ Voir [12] pour la théorie de Bohm et [13,14] pour la théorie GRW.

${ }^{4}$ Voir [15] pour une discussion ouverte entre plusieurs physiciens au sujet des raisons d'accepter ou de refuser la théorie de Bohm.
} 


\subsection{La position de Bohr et l'interprétation de Copenhague}

L'interprétation de Copenhague est principalement due à Bohr avec des contributions essentielles de Heisenberg et Born. On peut la décrire de manière approximative comme partant de l'idée que les objets macroscopiques ont des propriétés bien définies et n’obéissent pas à la mécanique quantique. On considère alors qu'une mesure est une interaction entre un système quantique et un appareil de mesure macroscopique, et que la propriété mesurée n’appartient pas au système seul mais à l'ensemble constitué par le système et l'appareil de mesure. Dans ce cas, un physicien utilisant un appareil macroscopique sait qu'il fait une mesure et doit appliquer le principe de réduction du paquet d'ondes. L'ambiguïté de choix avec l'équation de Schrödinger semble donc résolue. En fait, bien que cette position soit cohérente dans un cadre instrumentaliste, elle repose sur le qualificatif de macroscopique qu'il est impossible de définir précisément. Elle ne fait donc que repousser le problème si l'on veut être rigoureux. La position de Bohr est en fait plus subtile car il avait en tête des raisons pragmatistes. Mais nous nous contenterons ici d'indiquer que, bien que dominante pendant plusieurs décennies, cette position n'est plus guère soutenue que par les physiciens qui ne se préoccupent pas des problèmes de fondements du formalisme.

\subsection{Interprétations faisant intervenir la conscience de l'observateur}

Devant la difficulté d'éviter de mentionner l'observateur durant le processus de mesure, un certain nombre d'auteurs ont proposé que bien que la mécanique quantique ait une validité universelle, la conscience de l'observateur restait en dehors de son champ d'application. Comme le dit von Neumann [1] :

"Experience only asserts something like: an observer has made a certain (subjective) perception, but never such as: a certain physical quantity has a certain value."

"It is inherently entirely correct that the measurement or the related process of the subjective perception is a new entity relative to the physical environment and is not reducible to the latter."

"But in any case, no matter how far we calculate, [....], at some time we must say: and this is perceived by the observer. That is, we must always divide the world into two parts, the one being the observed system, the other the observer."

Et Wigner ajoute [16] :

"When is the measurement completed? We will see that [...] the measurement is completed only when we have observed its outcome"

Selon ces points de vue, une mesure n'est faite sur un système que lorsque la conscience d'un observateur a interagi avec lui. C'est cette interaction qui a pour effet de déterminer la valeur du résultat et de changer l'état du système. Cet effet est postulé par Wigner [17] et par London et Bauer [18]. Comme nous le verrons, ces auteurs eurent une intuition correcte concernant le fait que la conscience ou la perception de l'observateur ne peut être éliminée si on veut définir ce qu'est une mesure dans un cadre quantique standard mais ils en tirèrent une conclusion erronée. Postuler que la conscience échappe à une description physique mais peut cependant avoir une action sur la matière rappelle étrangement le dualisme cartésien qui est aujourd'hui rejeté de manière unanime. Nous verrons que le Solipsisme Convivial fait aussi jouer un rôle majeur à la conscience mais d'une manière toute différente et qui échappe à cette critique car la conscience n'a aucun effet sur la matière.

\subsection{Interprétations considérant le vecteur d'état comme relatif}

Je présente dans ce qui suit trois interprétations qui partagent le point commun de considérer que le vecteur d'état n'est pas absolu mais est relatif. Ceci sera aussi une caractéristique du Solipsisme Convivial. 


\subsubsection{L’interprétation relationnelle}

L'interprétation relationnelle est due à Rovelli qui nie que l'état d'un système est indépendant de l'observateur et dit qu'au contraire, un système n'a d'état que relativement à un autre système [19 - 21]. Rovelli appelle l'autre système un « observateur » mais il insiste sur le fait que cet autre système n'a nul besoin d'être un humain, d'être conscient ou même d'être macroscopique : un électron peut jouer le rôle d'un observateur vis à vis d'un autre électron. Mais Rovelli n'explique nulle part ce qu'une observation et une mesure sont. Il semble postuler le fait que toute interaction entre deux systèmes physiques (qui a pour conséquence que les deux systèmes s'intriquent, selon la conception usuelle) aboutit au fait que l'état de l'un des systèmes est réduit relativement au point de vue de l'autre (et vice versa). Cette conclusion étrange provient du fait qu'il semble confondre deux choses : le fait a) qu'après l'interaction entre $\mathrm{S}$ et $\mathrm{O}$, il y a une corrélation entre l'état de $\mathrm{S}$ et celui de $\mathrm{O}$ (par exemple si $\mathrm{S}$ est dans l'état $|1\rangle$ alors $\mathrm{O}$ est dans l'état $\left|O_{1}\right\rangle$ et si $\mathrm{S}$ est dans l'état $|2\rangle$ alors $\mathrm{O}$ est dans l'état $\left|O_{2}\right\rangle$ ), et le fait b) qu'une mesure a été faite ce qui aboutit à ce que $\mathrm{S}$ et $\mathrm{O}$ sont dans un état unique bien défini :

"The fact that the pointer variable in $O$ has information about $S$ (has measured q) is expressed by the existence of a correlation between the $q$ variable of $S$ and the pointer variable of $O$. The existence of this correlation is a measurable property of the S-O state". [19]

Cette corrélation à laquelle il fait allusion reflète simplement l'intrication entre les deux systèmes alors qu'une véritable mesure aboutit à une réduction à une seule des possibilités offertes, soit $\left|O_{1}\right\rangle|1\rangle$ soit $\left|O_{2}\right\rangle|2\rangle$. Assimiler une simple corrélation à une mesure est difficile à accepter ${ }^{5}$. Et si ce n'est pas le cas, on ne voit vraiment pas pourquoi toute interaction entre deux systèmes (même microscopiques) devrait être une mesure qui aboutit à sélectionner l'une des valeurs possibles. Cette solution du problème de la mesure n'est donc pas acceptable.

\subsubsection{Le Bayesianisme quantique ou QBism}

Le QBism est une interpretation issue du Bayésianisme quantique et elle est aujourd'hui principalement défendue par Fuchs, Schack and Mermin [22-25]. Les QBists pensent que l'objet central de la science est l'expérience individuelle. Pour eux, le vecteur d'état d'un système ne représente pas les propriétés objectives de ce système mais uniquement les probabilités subjectives qu'un agent attribue aux résultats des mesures potentielles qu'il pourrait faire sur le système. Le vecteur d'état est donc entièrement relatif à chaque agent. La mécanique quantique ne porte donc pas sur le réel mais sur la manière dont chaque agent peut quantifier ses propres attentes de résultats futurs. Dans ce cadre, une mesure est une action qu'un agent fait sur le monde extérieur. Il n'y a donc aucune mesure quand il n’y a pas d'agent. Le résultat de la mesure est l'expérience personnelle que l'agent récolte après son action. Le résultat de la mesure est donc lui aussi relatif à l'agent qui a fait la mesure. Il n'y a donc plus d'ambigüité sur le choix entre l'équation de Schrödinger et le principe de réduction puisque de toute façon, le vecteur d'état ne représente pas le système mais uniquement les croyances de l'agent sur le système. Le principe de réduction signifie seulement que ces croyances sont révisées lorsqu'un nouveau résultat est obtenu. Des points communs avec le Solipsisme Convivial sont d'une part, le fait que l'observateur joue un rôle essentiel dans la mesure et que d'autre part, on est conduit à renoncer à une description absolue de la réalité. Mais le QBism reste flou sur beaucoup de points importants qui demanderaient à être précisés pour pouvoir considérer que cette interprétation est totalement satisfaisante. On se référera à l'analyse détaillée que j’en donne dans [26].

\subsubsection{L'interprétation d'Everett}

Everett a explicitement développé son interprétation dans le but de résoudre le problème de la mesure qu'il considérait comme une véritable contradiction au sein de la mécanique quantique. En

\footnotetext{
${ }^{5}$ Rovelli prétend cependant que l'hypothèse selon laquelle un observateur est simplement un système physique ayant acquis de l'information (c’est à dire une corrélation) sur un autre système est au cœur de l'interprétation relationnelle [19].
} 
particulier, il trouvait le principe de réduction du paquet d'onde « répugnant ». Il proposa $[27,28]$ une formulation sans réduction, appelée interprétation des états relatifs, dans laquelle la fonction d'onde de l'univers évolue uniquement selon l'équation de Schrödinger. Ses idées furent ensuite popularisées par Graham et De Witt sous le nom de théorie des mondes multiples [29]. Pour rendre compte du fait que l'observateur voit bien un seul résultat de mesure, il est postulé que lors de chaque mesure, l'univers et l'observateur se scindent en autant d'exemplaires différents qu'il y a de résultats de mesure possibles. Donc tout ce qui est possible se produit et l'univers se ramifie en permanence en branches qui contiennent toutes les possibilités. Cette conception a évidemment suscité dès le début de nombreux commentaires négatifs mais étrangement, elle connait un regain récent de popularité malgré les nombreux problèmes qu'elle soulève. Un premier problème est celui de la prolifération exorbitante de mondes différents (en nombre infini continu). Mais cela pourrait être considéré comme une simple affaire de goût personnel. Un deuxième problème beaucoup plus difficile à résoudre est le fait que, comme tout ce qui est possible se produit, la notion de probabilité perd tout sens. La règle de Born que chaque observateur peut expérimenter doit alors trouver une explication. Mais toutes les tentatives pour réintroduire de manière cohérente la notion de probabilité dans ce cadre, se révèlent loin d'être convaincantes. Un certain nombre d'autres problèmes complémentaires que nous n'aborderons pas ici, font que cette théorie ne nous semble pas acceptable et c'est d'ailleurs en réaction à cette prolifération excessive de mondes que le Solipsisme Convivial a été initialement développé.

\section{Une synthèse des questions à résoudre}

A ce stade, il est utile de résumer les différentes questions, relatives aux fondements du formalisme, que toute interprétation cherche à résoudre (on pourra consulter [30] où ces question sont plus longuement justifiées et détaillées) :

- Qu'est-ce qu'une mesure et quand devons-nous utiliser l'équation de Schrödinger ou le postulat de réduction pour décrire l'évolution d'un système?

- Quand et pourquoi un seul des résultats possibles est-il obtenu ?

- Si une mesure ne peut être considérée comme révélant une valeur pré-existante, comment est-il possible que la valeur obtenue puisse-t-être créée lors de la mesure ?

- Cette valeur appartient-elle au système lui-même, au système et à l'appareil de mesure ou est-elle liée également à l'observateur?

- Concerne-t-elle la réalité extérieure ?

- Comment sait-on quelle propriété on mesure quand on utilise un appareil donné (c'est ce qu’on appelle communément le problème de la base préférée) ?

- Comment doit-on comprendre la non-localité montrée par la violation des inégalités de Bell et existet-il des actions à distance instantanées ?

Certaines des interprétations mentionnées ci-dessus répondent à une partie de ces questions mais aucune ne répond de manière satisfaisante à la totalité d'entre elles. Nous n'aurons pas ici le temps d'examiner en détail les deux dernières questions qui appelleraient des développements complémentaires mais nous verrons cependant que le Solipsisme Convivial permet de répondre à toutes. On pourra consulter [30, 34, 35, 36] pour des analyses plus détaillées de ces points ou [39] pour une présentation vulgarisée.

\section{Le Solipsisme Convivial}

Le solipsisme convivial est né dans le but de résoudre le problème de la mesure en évitant le principe de réduction du paquet d'ondes sous sa forme standard, comme dans l'interprétation d'Everett mais sans pour autant devoir accepter l'existence d'innombrables mondes parallèles. Bien sûr, la majorité des physiciens préféreraient résoudre les problèmes des fondements de manière totalement objective, c'està-dire en se passant de mentionner l'observateur. Mais il semble bien, qu'à moins d'accepter de modifier 
le formalisme standard, aucune tentative dans ce sens ne soit satisfaisante. Il est donc intéressant d'explorer les possibilités qu'offre le fait de faire jouer un rôle à l'observateur. Cette direction n'est pas nouvelle. Nous avons déjà mentionné le rôle que von Neumann et Wigner voulaient faire jouer à la conscience mais même Planck disait :

"I regard consciousness as fundamental. I regard matter as derivative from consciousness. We cannot get behind consciousness. Everything that we talk about, everything that we regard as existing, postulates consciousness." 6

En fait, il est facile de voir que le problème de la mesure survient essentiellement dans un cadre réaliste où le vecteur d'état représente l'état physique du système indépendamment de tout observateur et où il est donc le même pour tous. Si en revanche, le vecteur d'état est relatif à chaque observateur et s'il est clair que le postulat de réduction ne traduit que le fait qu'un observateur devient conscient d'un résultat donné, alors le problème disparaît comme nous allons le voir.

Comme le dit Bitbol [31] :

"The measurement problem boils down to finding a way to articulate the indefinite chain of relational statements of the quantum theory to the absolute statements that are used in the experimental work. An articulation of this kind can easily be found, provided one realizes that the latter absolute statements are in fact indexical; provided one realizes that these statements are only absolute relative to us [...]. At this point one is bound to realize the ineliminability of situatedness from the apparent neutral descriptions of quantum mechanics."

Le Solipsisme Convivial ${ }^{7}$ tire toutes les conséquences de ces idées dans une perspective néokantienne. Il suppose que la dynamique d'évolution de l'univers est décrite par l'équation de Schrödinger et qu'une mesure n'est que la perception d'un des résultats possibles par un observateur. L'idée principale est qu'en raison des limites perceptives humaines, une fonction d'onde superposée ne peut être perçue comme telle par un observateur. En conséquence, un observateur qui voit un système dans un tel état superposé le percevra à travers certains filtres mentaux qui lui donneront l'impression d'observer un résultat bien défini et que le système est lui aussi dans un état macroscopiquement bien défini. Mais ce qu'il verra n'est que le résultat de ses limites perceptives et le système en lui-même ne changera pas d'état et restera dans un état superposé. Il n’y a donc aucune réduction physique du paquet d'ondes car la projection prédite par ce postulat n'est que le reflet de ce que peut percevoir l'observateur. Elle n’a aucune réalité physique.

On peut donner une idée intuitive de ce qui se passe par analogie avec certaines images qu'il est possible de voir de différentes manières. L'une des plus illustratives est l'image animée d'une danseuse que l'on peut voir tourner sur elle-même tantôt dans le sens des aiguilles d'une montre tantôt dans le sens inverse ${ }^{8}$. C'est une illusion d'optique bistable. Selon l'observateur qui la regarde, la danseuse semble danser dans le sens des aiguilles d'une montre ou dans le sens inverse. Se demander dans quel est sens réel tourne la danseuse n’a pas de sens. Il n’existe pas de direction réelle de rotation car cette image est simplement un ensemble de pixels en mouvement que notre cerveau interprète comme une danseuse tournant dans un sens ou dans l'autre. Ce n'est pas une image de danseuse tournant au sens propre du terme. En y mettant toutes les précautions nécessaires, on pourrait comparer l'état de cette danseuse avec une superposition :

$$
\mid \text { sens des aiguilles d'une montre }\rangle+\mid \text { sens inverse des aiguilles d'une montre }\rangle
$$

\footnotetext{
${ }^{6}$ Je remercie Chris Fuchs de m’avoir signalé cette citation de Planck dans un journal.

${ }^{7}$ Le Solipsisme Convivial est une version largement modifiée et étendue d'un modèle initialement proposé par d’Espagnat [32, 33]. J'en ai exposé une première version dans un livre en 2000 [34].

8 https://www.youtube.com/watch?v=9CEr2GfGilw
} 
Cet état ne correspond ni à tourner dans un sens ni à tourner dans l'autre mais représente quelque chose qui n'a pas d'équivalent classique. Quand nous regardons une telle image en revanche, nous ne sommes capables que de voir une danseuse qui tourne dans un sens déterminé. De plus, ce sens n'est pas nécessairement le même pour tous les observateurs. Mais voir la danseuse tourner dans un sens déterminé n'implique nullement que la danseuse tourne réellement dans ce sens et bien sûr, l'image en elle-même n'est pas modifiée et reste une superposition. Comme nous sommes incapables de percevoir deux sens de rotation superposés notre cerveau en sélectionne un au hasard. Il y a bien sûr de nombreuses différences entre un état quantique superposé et l'état de la danseuse mais cela permet d'avoir une idée approximative de ce qui se passe lorsqu’on perçoit un état superposé.

Formellement, le Solipsisme Convivial repose sur deux hypothèses principales : le mécanisme d'accrochage qui définit ce qu'est une mesure et la relativité des états quantiques.

\subsection{Première hypothèse : le mécanisme d’accrochage}

Mécanisme d'accrochage : Une mesure est la prise de conscience d'un résultat par un observateur dont la perception sélectionne aléatoirement (en accord avec la règle de Born) l'une des branches du vecteur d'état intriqué (avec l'appareil de mesure et l'environnement) du système, écrit dans la base préférée donnée par le mécanisme de décohérence. Nous appellerons cette sélection l'accrochage à la branche. Une fois que la perception est accrochée à une branche, elle ne pourra s'accrocher qu'à des branches filles de cette branche lors de toute observation ultérieure.

La dernière partie du mécanisme d'accrochage ${ }^{9}$ garantit que si on répète la même mesure, on obtiendra le même résultat. Prenons l'exemple de la mesure d'une particule de spin $1 / 2$ dans un état superposé suivant $\mathrm{Oz}$ :

$$
\left|\Psi_{S}\right\rangle=\alpha|+\rangle_{z}+\beta|-\rangle_{z}
$$

Après l’interaction avec l'appareil, l'état devient:

$$
\left|\Psi_{S A}\right\rangle=\alpha|+\rangle_{z}|\uparrow\rangle+\beta|-\rangle_{z}|\downarrow\rangle
$$

Et si on inclue l'état physique d'un observateur regardant l'appareil :

$$
\left|\Psi_{S A O}\right\rangle=\alpha|+\rangle_{z}|\uparrow\rangle|\odot\rangle+\beta|-\rangle_{z}|\downarrow\rangle|\odot\rangle
$$

où $|\uparrow\rangle$ (resp. $|\downarrow\rangle)$ est l'état de l'appareil corrélé au spin + (resp. spin -) and $|\odot\rangle$ (resp. $|\otimes\rangle$ ) est l'état physique du cerveau de l’observateur corrélé à l'état $|\uparrow\rangle$ (resp. $|\downarrow\rangle)$ de l'appareil.

Maintenant le mécanisme d'accrochage stipule que la perception de l'observateur sélectionne une branche. Notons $\widetilde{-}$; le fait d'être conscient d'avoir perçu “+” (resp. by $\widetilde{*}$ avoir perçu “-”). Après accrochage, soit l'observateur aura vu “+” soit il aura vu “-”. Donc après la mesure, la fonction d'onde du système global sera toujours (7) mais l'état de perception de l'observateur sera $\widetilde{0}$ ou $\widetilde{\otimes}$. Il est important de faire une différence entre l'état physique du cerveau de l'observateur et sa perception. $|\odot\rangle$ and $|:\rangle$ sont les états physiques du cerveau de l'observateur et sont des kets entrant dans la fonction d'onde superposée. Alors que $\widetilde{\odot}$ et $\widetilde{*}$ ) ne sont pas des kets et ne sont nullement les composantes d'une fonction d'ondes. Ils décrivent simplement des états de perception. Bien entendu, l'état de perception $\widetilde{\odot}$ est associé à l'état physique du cerveau $|-()\rangle$ au sens où si le cerveau est dans l'état physique $|\odot\rangle$ alors la perception est nécessairement $\widetilde{\odot}$; (et idem pour l'autre état).

Selon le mécanisme d'accrochage, une fois une branche sélectionnée, il ne sera possible à la perception de l'observateur que de s'accrocher à des branches filles de la première, lors des mesures ultérieures. Donc, même si la fonction d'onde globale reste la fonction superposée (7), tout se passera

\footnotetext{
${ }^{9}$ En fait, le mécanisme d'accrochage est plus complexe que cela si on prend en compte la décohérence et la relativité des états, mais cela ne change pas la présentation simplifiée faite ici. Voir [30,35,36] pour une analyse plus détaillée.
} 
pour l’observateur comme si la fonction d'onde était réduite à $|+\rangle_{z}|\uparrow\rangle|\odot\rangle$ ou à $|-\rangle_{z}|\downarrow\rangle|\otimes\rangle$ selon que son état de perception est $: ;$ ou $: *$. Ceci garantit que répéter la même mesure fournit bien le même résultat.

Contrairement à ce qui se passe avec l'interprétation d'Everett, il n’y a ici aucune difficulté avec les probabilités puisque, exactement comme en mécanique quantique standard, la règle de Born est explicitement utilisée par le mécanisme d'accrochage, ce qui permet de rendre compte des probabilités que nous observons. En revanche, les probabilités ne sont plus associées au phénomène physique de réduction (qui n'existe plus) mais à la sélection faite par la conscience de la branche à laquelle elle s'accroche. En réponse à la fameuse phrase d'Einstein qui, refusant le hasard objectif en mécanique quantique avait dit «Dieu ne joue pas aux dés », on peut dire que c'est l'observateur qui y joue !

Une autre caractéristique essentielle est que pour un observateur donné, les autres observateurs sont des systèmes physiques comme n'importe quel autre système physique. Ils peuvent donc être également dans des états superposés et s'intriquer avec les autres systèmes. En revanche, un observateur ne peut avoir aucun accès direct aux perceptions d'un autre observateur. Les seules informations qu'il peut connaitre au sujet des perceptions d'un autre doivent être communiquées par un moyen physique quelconque (la parole, la vision, l'écriture etc ...). Cela sera fondamental pour expliquer la raison pour laquelle il ne peut y avoir de divergence entre deux observateurs au sujet du résultat d'une même mesure.

\subsection{Deuxième hypothèse : la relativité des états}

Tout ce qui vient d'être dit pourrait être compris comme s'il y avait une réalité indépendante unique dont tous les observateurs sont témoins et qui est décrite par une unique fonction d'ondes globale enchevêtrée. Bien entendu, compte tenu du mécanisme d'accrochage qui s’applique indépendamment à chaque observateur, chacun possèderait sa propre perception de cette réalité mais il y aurait une seule fonction d'onde globale. C'est ce qui se produit dans la conception d'Everett. Mais le Solipsisme Convivial partage avec le QBism et l'interprétation relationnelle de Rovelli l'idée que toutes les entités du formalisme sont en fait relatives (à un système dans le cas de l'interprétation relationnelle, à un agent dans le cas du QBism et à un observateur dans le cas du Solipsisme Convivial). Le renoncement à une description absolue de la réalité est donc encore plus fort puisque même la description formelle sur laquelle repose les possibilités de prédiction des perceptions de l'observateur lors d'une mesure qu'il effectuera, est propre à cet observateur. L'univers n'est donc plus une entité absolue existant par ellemême indépendamment de tout observateur mais devient une description relative à chaque observateur. Comme je l'explique dans [30] :

"Convivial Solipsism is situated in a neo-Kantian framework and assumes that there is "something" else than consciousness, something that (according to the famous Wittgenstein's sentence) it is not appropriate to talk of. This is close to what Kant calls "thing in itself" or "noumenal world". Consciousness and this "something" give rise to what each observer thinks is her reality, following Putnam's famous statement "the mind and the world jointly make up the mind and the world". So perception is not a passive affair: perceiving is not simply witnessing what is in front of us but is creating (independently for each us) what we perceive through a co-construction from the world and the mind. The hanging-on mechanism takes part in this co-construction and helps (very partially) understanding it through the selection it does."

Cette relativité des états a une conséquence fondamentale sur le langage autorisé par le Solipsisme Convivial. Toute phrase doit être prononcée du point de vue d'un interlocuteur unique et rapportée à sa propre perception. En particulier, il est interdit de parler simultanément des perceptions de deux observateurs différents. Seul un meta-observateur pourrait avoir accès aux perceptions d'autres observateurs et un tel meta-observateur (qu'on appelle quelquefois le point de vue de Dieu) n'existe pas. La phrase « Alice a vu ceci et Bob a vu cela » n’est donc pas autorisée car elle se réfère directement aux perceptions simultanées de deux observateurs différents. En revanche, il est possible de dire « Alice a 
vu ceci et a entendu Bob lui dire qu'il a vu cela » qui est une phrase qui se réfère au point de vue d'Alice uniquement. En effet, la perception de Bob n’est mentionnée qu’à travers la perception qu'Alice en a eue par la parole.

\subsection{Pourquoi le Solipsisme Convivial est-il convivial ?}

Puisque le mécanisme d'accrochage est relatif à chaque observateur, on peut se demander s'il peut en résulter un conflit entre deux observateurs qui divergeraient sur ce qu'ils ont observé (comme c'est le cas pour la danseuse que nous avons vue plus haut). La réponse est que cela ne peut se produire et c'est la raison pour laquelle cette interprétation est qualifiée de conviviale alors même qu'on a noté le fait que les perceptions de chaque observateur lui sont propres. On peut déjà remarquer que conformément à ce qui vient d'être dit, une phrase comme "Sachant qu'Alice a vu le spin "+" et que Bob a vu le spin "-", qu'entendra Alice si elle interroge Bob ? » n’est pas autorisée car elle mentionne simultanément les perceptions de Alice et Bob. En revanche, on a le droit de s'interroger pour savoir si Alice ayant vu "+", elle peut entendre Bob lui dire qu'il a vu "-". La réponse est que cela est impossible en raison du fait que toute communication entre deux observateurs est en fait une mesure de l'un sur l'autre. Comme le dit d'Espagnat [33] :

"Any transfer of information from B to A-for example, any answer made by $B$ to a question asked by A- unavoidably proceeds through physical means. Therefore it necessarily takes the form of a measurement made by $A$ on $B$. And we know that under these conditions A necessarily gets a response (answer) that agrees with his own perception”.

Alice ayant fait une mesure et étant accrochée à la branche correspondant au résultat "+" ne peut s'accrocher qu'à des branches filles de cette branche pour toute mesure ultérieure. Bob (considéré comme un simple système physique) est dans un état intriqué avec le spin du système mais lorsqu'Alice l'interroge, elle mesure Bob et ne peut obtenir come résultat que celui qui provient de la composante de l'état de Bob qui est corrélée au spin "+". Il en résulte qu'Alice entendra bien Bob lui dire qu'il a vu "+" conformément à ce qu'elle a elle-même perçu. Aucun conflit n’est donc possible. En revanche, Alice doit éviter d'en conclure que Bob a "réellement » vu "+" car elle ne peut avoir aucun accès aux perceptions de Bob et bien sûr elle doit encore plus éviter de conclure que le spin était « réellement » "+".

Ce processus de communication entre deux observateurs est important car il est la clé pour comprendre comment le Solipsisme Convivial évite la non-localité.

\subsection{Comment éviter la non-localité ?}

On supposera connu du lecteur l'argument d'Einstein, Podolsky et Rosen [37] et les inégalités de Bell [38] qui conduisent en général à la conclusion que toute théorie faisant les mêmes prédictions que la mécanique quantique est non-locale. Le raisonnement repose sur l'examen de deux particules $\mathrm{U}$ et $\mathrm{V}$ intriquées dans un état singulet de spin du type :

$$
|\psi\rangle=\frac{1}{\sqrt{2}}\left[|+\rangle^{U}|-\rangle^{V}-|-\rangle^{U}|+\rangle^{V}\right]
$$

Selon la présentation traditionnelle, lorsqu'une mesure du spin selon une direction est faite sur l'une des particules à un endroit donné, son état est projeté sur l'un des deux états possibles et l'autre particule voit également son état projeté sur l'état corrélé correspondant et ce, instantanément et quelle que soit la distance entre les deux particules. Cette corrélation qui intervient même lorsqu'un intervalle du genre temps sépare les deux mesures, semble dont faire intervenir une action non-locale. Cette conclusion vient du fait que la mécanique quantique interdit de penser que l'état des particules est déterminé avant la mesure et les inégalités de Bell dont on a montré expérimentalement qu'elles sont violées, interdisent de considérer que des variables cachées locales peuvent expliquer les corrélations à distance qui sont observées. 
Mais ce raisonnement repose sur la corrélation obtenue entre deux mesures (celle faite par un observateur sur la première particule à un endroit donné et celle faite par un autre observateur sur l'autre particule à un autre endroit). Dans le raisonnement traditionnel, il parait évident qu'il est possible de parler de manière absolue de ces deux résultats comme de faits objectifs et pouvant s'être produits simultanément. D’où la conclusion de non-localité. Mais il n'en est rien dans le Solipsisme Convivial puisque nous l'avons vu, toute mesure est relative à un observateur unique. Les corrélations obtenues (qui seront bien vérifiées) ne pourront l'être qu'en deux temps. Un observateur fera une mesure sur une particule puis pour constater la corrélation avec l'autre résultat, il devra interroger ultérieurement l'autre observateur et connaître ainsi le deuxième résultat. Mais dans le cadre de cette interprétation, comme aucun changement physique ne se produit lors d'une mesure, cette procédure doit seulement s'interpréter comme le fait qu'une première mesure donne pour un observateur un résultat qui est bien corrélé avec le résultat que le même observateur obtiendra quand il interrogera le deuxième observateur. Bien entendu, cette interrogation ne pourra se produire que dans un intervalle du genre espace avec la première mesure et il n'y aura donc plus aucune non-localité (voir [36] pour un raisonnement plus approfondi sur la non-localité).

\section{En résumé}

Il est maintenant possible de jeter un regard en arrière et de comprendre les points positifs des différentes interprétations qui ont été proposées antérieurement. Chacune contenait un élément positif.

- L'interprétation de Copenhague insistait à juste titre sur l'expérience et sur l'aspect contextuel de la mesure mais reposait sur une distinction microscopique / macroscopique ambigüe et ne reconnaissait pas pleinement le rôle de l'observateur.

- Wigner, von Neumann, London et Bauer faisaient remarquer qu'il est impossible de donner une interprétation cohérente de la mécanique quantique sans mentionner la conscience de l'observateur. Mais ils attribuaient à cette conscience une action sur la matière qui n'est pas acceptable.

- Everett avait correctement énoncé le fait que la fonction d'onde globale de l'univers n'est jamais réduite. Mais dans son interprétation, d'une part, la notion de probabilité perd son sens et d'autre part, la multiplication de mondes parallèles à laquelle le conduit son explication de la raison pour laquelle un observateur voit un résultat unique n’est pas satisfaisante.

- L'interprétation relationnelle admet l'aspect relatif des vecteurs d'état mais sa conception de la mesure est incohérente.

- Le QBism bien que clarifiant le problème de la mesure laisse beaucoup trop de questions ouvertes concernant son ontologie et est incapable d'expliquer l'apparence classique d'un monde qui reste quantique dans son essence.

Le Solipsisme Convivial donne des réponses satisfaisantes à toutes les questions évoquées au paragraphe 4. Seule la réponse à la sixième question portant sur le choix de la base préférée n’a pas été détaillée dans ce texte mais la réponse provient de l’utilisation faite de la décohérence. Pour plus de précisions on se référera à [30, 34, 35, 36, 39].

Bien sûr, l’image donnée par le Solipsisme Convivial est très loin des conceptions réalistes traditionnelles. La réalité n'est plus la même pour tous les observateurs qui, d'une certaine manière, vivent chacun dans leur propre monde de perceptions. C'est en ce sens que cette interprétation peut être considérée comme solipsiste car il n'existe pas de réalité commune. Mais ce n’est pas un solipsisme au sens fort du terme puisqu'il y est admis non seulement l'existence d'autres observateurs mais aussi celle d'une réalité indépendante. Par ailleurs, comme aucune divergence ne peut apparaître entre les différents observateurs, le qualificatif de convivial peut lui être appliqué. Le prix à payer est aussi d'accepter l'impossibilité de parler directement des perceptions d'un autre observateur que celui qui s'exprime. 
Mais la physique nous a habitué à renoncer progressivement aux notions qui nous paraissaient les plus intuitives. Qu'on se rappelle la difficulté avec laquelle l'abandon du concept d'évènements simultanés a été accepté.

\section{Références}

1. Von Neumann, J.: Mathematische Grundlagen der Quanten-mechanik, Springer-Berlin (1932). English translation: Mathematical Foundations of Quantum Mechanics, Princeton University Press (1955)

2. Schrödinger, E.: Die gegenwärtige Situation in der Quantenmechanik (The present situation in quantum mechanics). Naturwissenschaften 23 (1935)

3. Wigner, E.P.: Remarks on the mind-body question. In: I.J. Good. The Scientist Speculates. London, Heinemann (1961)

4. Hacking, I.: Representing and Intervening. Cambridge University Press (1983)

5. Pickering, A.: The Mangle of Practic. Chicago University Press (1995)

6. Bachtold, M. : L’interprétation de la Mécanique Quantique. Une approche pragmatiste. Hermann (2008)

7. Healey, R.: Quantum Theory: a Pragmatist Approach. British Journal for Philosophy of Science, 63 (2012)

8. Bell, J.S.: On the problem of hidden variables in quantum mechanics. Rev. Mod. Phys., 38, (1966)

9. Bohm, D.: A suggested interpretation of the quantum theory in terms of 'hidden' variables. I. Physical Review, 85: 166-179; II. Physical Review, 85: 180-193 (1952)

10. Ghirardi, G.C., Rimini, A., Weber, T.: Phys. Rev., D34, 470 (1986)

11. Ghirardi, G.C.: Collapse Theories. In: The Stanford Encyclopedia of Philosophy (2011)

12. Dürr, D., Goldstein, S., Norsen, T., Struyve, W., Zanghì, N.: Can Bohmian mechanics be made relativistic?. Proceedings of the Royal Society A 470, doi:10.1098/rspa.2013.0699 (2013). Preprint arXiv:1307.1714 (2013)

13. Tumulka, R.: A Relativistic Version of the Ghirardi-Rimini-Weber Model. Journal of Statistical Physics. 125 : 821 (2006)

14. Bedingham, D.: Towards Quantum Superpositions of a Mirror: an Exact Open Systems Analysis. Journal of Physics. A38: 2715 (2011)

15. d’Espagnat, B., Zwirn, H. : Le monde quantique. Les débats philosophiques de la mécanique quantique. Editions Matériologiques (2014)

16. Wigner E. P.: Interpretation of quantum mechanics. (1976) In: Quantum Theory and Measurement. Wheeler J. A., Zurek W. (eds.) Princeton University Press (1983)

17. Wigner, E.P.: Symetries and Reflections. Bloomington, Indiana University Press (1967)

18. London, F., Bauer, E.: La théorie de l’observation en mécanique quantique. Hermann (1939)

19. Rovelli, C.: Relational Quantum Mechanics. International Journal of Theoretical Physics. 35, 16371678 (1996)

20. Rovelli, C., Smerlak, M.: Relational EPR. Foundations of Physics. 37 (2007)

21. Laudisa, F., Rovelli, C.: Relational quantum mechanics. In: The Stanford Encyclopedia of Philosophy (2008)

22. Fuchs, C.A.: 'Quantum Bayesianism at the perimeter'. arXiv: 1003:5182 (2010)

23. Fuchs, C.A.: ‘On participatory Realism’. arXiv.org/abs/1601.04360 (2016)

24. Fuchs, C.A.: 'Notwithstanding Bohr, the Reasons for QBism’. arXiv: 1705.03483 (2018)

25. Fuchs, C.A., Mermin, D.N. \& Schack, R.: 'An Introduction to QBism with an application to the locality of quantum mechanics’, American Journal of Physics, vol. 82, p.749. arXiv: 1311:5253. (2014)

26. Zwirn, H.: Is QBism aPossible Solution to the Conceptual Problems of Quantum Mechanics?, arXiv: 1912.11636, (2019) to appear in the forthcoming Oxford Handbook of the History of Interpretations and Foundations of Quantum Mechanics.

27. Everett, H.: On the Foundations of Quantum Mechanics. Ph.D. thesis. Princeton University, Department of Physics (1957)

28. Everett, H.: Relative State Formulation of Quantum Mechanics. Reviews of Modern Physics. 29: 454-462 (1957)

29. DeWitt, B. S., Graham N. (eds.): The Many-Worlds Interpretation of Quantum Mechanics. Princeton: Princeton University Press (1973) 
30. Zwirn, H.: ‘The Measurement Problem: Decoherence and Convivial Solipsism’, Found. Phys. Vol. 46, p.635. arXiv:1505.05029 (2016)

31. Bitbol, M.: Reflective Metaphysics: Understanding Quantum Mechanics from a Kantian Standpoint. Philosophica 83 (2010)

32. d’Espagnat, B.: Le Réel voilé, analyse des concepts quantiques. Fayard (1994). English Transl: Veiled Reality: An Analysis of Quantum Mechanical Concepts. Westview Press, Boulder, Colorado (2003)

33. d’Espagnat, B.: Conceptual Foundations of Quantum Mechanics. Benjamin, New York (1971)

34. Zwirn, H.: 'Les limites de la connaissance', Odile Jacob (Ed) (2000)

35. Zwirn, H.: 'Delayed Choice, Complementarity, Entanglement and Measurement', Physics Essays, Vol. $30,3$. arXiv:1607.02364 (2017)

36. Zwirn, H.: 'Non Locality versus Modified Realism’, Found. Phys. Vol. 50, pp 1-26. arXiv:11812.06451 (2020)

37. Einstein, A., Podolsky, B., Rosen, N.: Can Quantum-Mechanical Description of Physical Reality Be Considered Complete?. Physical Review. 47: 777-780 (1935)

38. Bell, J.S.: On the Einstein-Podolski-Rosen Paradox. Physics. 1 (1964)

39. Zwirn, H.: ‘Le rôle de l’observateur en mécanique quantique’, Pour la Science, mars 2020, num. 509, (2020) 